\title{
TITLE:
}

\section{Tunnel numbers of knots and Jones-Witten invariants}

$\operatorname{AUTHOR}(\mathrm{S})$ :

KOHNO, TOSHITAKE

CITATION:

KOHNO, TOSHITAKE. Tunnel numbers of knots and Jones-Witten invariants. 数理解析研究所講究録 1993, 840: 1-19

ISSUE DATE:

1993-05

URL:

http://hdl.handle.net/2433/83536

RIGHT: 


\section{Tunnel numbers of knots and Jones-Witten invariants TOSHITAKE KOHNO \\ Department of Mathematical Sciences \\ University of Tokyo \\ Komaba, Tokyo 153, Japan

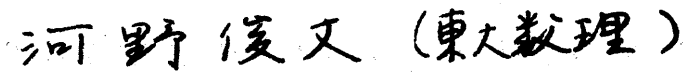

\section{Introduction and main results}

The purpose of this paper is to discuss some topological consequences derived from Jones-Witten invariants for links in 3-manifolds. In [Jo], Jones obtained upper bounds for the values of the Jones polynomial at roots of unity in terms of the braid index. In the present paper we focus on classical invariants called the tunnel numbers of knots. Let $K$ be a knot in $S^{3}$. We are going to describe a lower bound for the tunnel number $t(K)$ by means of special values of the Jones polynomial and its generalizations. The tunnel number is the minimal number of arcs $\gamma_{1}, \cdots, \gamma_{t}$ so that the closure of the complementary space of the regular neighborhood of $K \cup \gamma_{1} \cup \cdots \cup \gamma_{t}$ is homeomorphic to a handlebody. In Figure 1 we indicate that the tunnel number of a trefoil knot is equal.to one. It is clear that the tumnel number is bounded from above the minimal number of crossings of the knot diagram and that the minimal number of generators of the fundamental group of the knot complement is at most $t(K)+1$. This definition of the tunnel number is also generalized in a natural way to a knot in a closed oriented 3-manifold. In the preceding article [Ko3], we developed a general framework of giving an estimate for the tunnel number in terms of the Jones-Witten invariants, based on the monodromy of conformal field theory. The scope of the present paper is to give a refinement of the inequality obtained in [Ko3] and to present a more combinatorial description using quantum $6 j$-symbols at roots of unity.

To state our main results let us recall some basic construction for invariants of framed links associated with the quantum group $U_{q}(s l(2, \mathrm{C}))$ in the sense of Drinfel'd [D] and Jimbo [J]. We fix a positive integer $l$ called a level and we put $r=l+2$. In this paper we discuss the invariants at $q=e^{2 \pi \sqrt{-1} / r}$. Let $P_{+}(l)$ be a set of half integers defined by

$$
P_{+}(l)=\{0,1 / 2,1, \cdots, l / 2\} \text {. }
$$

Here $j \in P_{+}(l)$ corresponds to an irreducible $U_{q}(s l(2, \mathrm{C}))$ module of dimension $2 j+1$. Let $L$ be a framed link in $S^{3}$ with $m$ components. As was constructed in KirillovReshetikhin $[\mathrm{KR}]$, for $\lambda:\{1, \cdots, m\} \rightarrow P_{+}(l)$, we can associate an invariant of

Partially supported by Grant-in-Aid for Scientific Rescarch on Priority Arcas 231

"Infinite Analysis." 
oriented framed link denoted by $J_{r}(L, \lambda)$. In this paper, we follow a normalization due to Kirby-Melvin [KM]. If $\lambda \equiv 1 / 2$, then $J_{r}(L, 1 / 2)$ is a variant of the Jones polynomial given by

$$
J_{r}(L, 1 / 2)=\left(2 \cos \frac{\pi}{r}\right) t^{3 L \cdot L} V_{L}\left(e^{-\frac{2 \pi \sqrt{-1}}{r}}\right)
$$

with $t=e^{\pi \sqrt{-1} / 2 r}$ where $V_{L}$ is the original Jones polynomial normalized as in [Jo]. In the above expression the symbol $L \cdot L$ is the sum of the sum of the entries of the linking matrix $\sum_{i j} l k\left(L_{i}, L_{j}\right)$, where $l k\left(L_{i}, L_{i}\right)$ is the framing on the component $L_{i}$. In the case of a knot $K$ assigned with a color $j \in P_{+}(l)$, we have the following description. Putting for an integer $n=0,1, \cdots, l, J_{K, n}=J_{r}(K, n / 2)$, we have a recursive formula

$$
J_{K, n}=\sum_{i=0}^{[n / 2]}(-1)^{i}\left(\begin{array}{c}
n-i \\
i
\end{array}\right) J_{K^{n-2 i}, 1}
$$

where $K^{\gamma}$ stands for the $\gamma$ parallel push offs defined by means of the framing. Let us notice that $\left|J_{r}(K, j)\right|$ does not depend on the framing on $K$. For $i, j \in P_{+}(l)$ we put

$$
S_{i j}=\sqrt{\frac{2}{r}} \sin \frac{(2 i+1)(2 j+1) \pi}{r} .
$$

With this notation we have the following.

Theorem A. Let $K$ be a knot in $S^{3}$. Then, the tunnel number $t(K)$ satisfies

$$
\sum_{j \in P_{+}(l)}\left|J_{r}(K, j)\right|^{2} \leq 2^{-t(K)} S_{00}^{-2(t(K)+1)},
$$

which gives a lower estimate for the tunnel number.

Let us recall that we have a symmetry

$$
\left|J_{r}(K, j)\right|=\left|J_{r}(K, l / 2-j)\right|
$$

obtained in $[\mathrm{KM}]$. Based on this, we can deduce from Theorem $\dot{A}$ that

$$
\left|J_{r}(K, j)\right|^{2} \leq\left(\frac{1}{2 S_{00}^{2}}\right)^{t(K)+1}-1
$$

for any $j \in P_{+}(l)$ such that $j \neq 0, l / 2, j \neq l / 2-j$.

In [W], Witten introduced a series of invariants for a link in 3-manifold based on quantum field theory with Chern-Simons action. A Dehn surgery formula of the Witten invariant for $s l(2, \mathrm{C})$ was studied extensively by Reshetikhin-Turaev [RT] and 
Kirb--Melvin [KM]. Let $K$ be a knot in a closed oriented 3-manifold $M$ assigned with $j \in P_{\div}(l)$. We suppose that there is a framed link $L$ in $S^{3}$ disjoint from $K$ and $(M, K)$ is obtained by the Dehn surgery on the framed link $L$. Let us denote by $m$ the number of components of $L$. The invariant we are going to investigate is

$$
Z_{r}(M, K ; j)=C^{s i g n(L)} \sum_{\lambda} S_{0 \lambda(1)} \cdots S_{0 \lambda(m)} J_{r}(K \cup L, j \cup \lambda)
$$

where the sum is for any $\lambda:\{1, \cdots, m\} \rightarrow P_{+}(l), C=\exp (-3(r-2) \pi \sqrt{-1} / 4 r)$, and $s: g n(L)$ denotes the signature of the linking matrix for $L$. In particular, in the case the knot $K$ is empty, we obtain an invariant of 3-manifold $Z_{r}(M)$. Let us note that we normalize the invariant so that $Z_{r}\left(S^{3}\right)=1$, in contrast to the Witten's original normalization. Our result for the tunnel numbers of knots in 3-manifolds is the following.

Theorem B. Let $M$ be a closed oriented 3-manifold and let $K$ be a knot in $M$. Then, by means of the Witten invariant, the tunnel number $t(K)$ has a lower estimate

$$
\sum_{j \in P_{+}(l)}\left|Z_{r}(M, K ; j)\right|^{2} \leq S_{00}^{-2(t(K)+1)}
$$

Let $\Sigma$ be a closed oriented surface of genus $g$ and we denote by $\mathcal{M}_{g}$ its mapping class group. To prove our main resul: we describe a projective unitary representation

$$
\rho: \mathcal{M}_{g} \rightarrow G L\left(\mathcal{H}_{\Sigma}\right)
$$

constructed using an idea due to Moore-Seiberg [MS] and Reshetikhin-Turaev [RT]. By means of this representation we show

$$
Z_{r}(M, K ; j)=S_{00}^{-g}<v_{j}^{*}, \rho(h) v_{0}>
$$

with some $v_{0}, v_{j} \in \mathcal{H}_{\Sigma}$ if the knot exterior admits a splitting

$$
E(K)=\operatorname{cl}(V-N(K)) \cup_{h}(-V)
$$

with a handlebody $V$ of genus $g$. Here $N(K)$ is a tubular neighborhood of $K, c l$ stands for the closure, and $h: \partial V \rightarrow \partial V$ is a glueing map. The equality holds when the glueing map is identity. Theorem $B$ is a consequence of the unitarity of $\rho$, and Theorem $\mathrm{A}$ is shown as a special case $M=S^{3}$ with an improvement using the symmetry derived from the Dynkin diagram automorphism.

Let us observe that in the case $K$ is empty the unitarity implies

$$
\left|Z_{\tau}(M)\right| \leq S_{00}^{-g}
$$


which gives a lower bound for the Heegaard genus of $M$. The inequality 1.8 has been obtained in [Wa], [Ko2] and [G].

The above interpretation of the Jones-Witten invariants in terms of the mapping class group was studied in [Kol] and independently in [C] and [CLM]. Even in the case of knots in $S^{3}$ the above 3-dimensional point of view plays an essential role to show our inequality, which reveals a close relationship between the special values Jones polynomial and the quantum field theory.

Let us also recall that the topological meaning of the values of the Jones polynomial at certain roots of unity is well studied (see [Jo]). Let $M_{L}$ be the 2-fold branched covering of $S^{3}$ branched along a link $L$. In the case $r=6$, it is known by Lipson [Lp] and Lickorish-Millet [LM] that

$$
\left|V_{L}\left(e^{2 \pi \sqrt{-1} / 6}\right)\right|=3^{d / 2}
$$

where $d=\operatorname{dim} H_{1}\left(M_{L}, \mathrm{Z} / 3 \mathrm{Z}\right)$. Hence the inequality 1.5 implies in this case

$$
2 \cdot 3^{d(K)+1}+\left|J_{r}(K, 1)\right|^{2} \leq 2 \cdot 6^{t(K)+1}-2
$$

with $d(K)=\operatorname{dim} H_{1}\left(M_{K}, \mathrm{Z} / 3 \mathrm{Z}\right)$ for $r=6$. A numerical computation in the case $r=5$ shows that if a knot $K$ satisfies

$$
\left|V_{K}\left(e^{2 \pi \sqrt{-1} / 5}\right)\right|>2.1489 \ldots
$$

then we have $t(K) \geq 2$. Although we could not get an inequality sharper than this, we can derive several interesting consequences (see Remark 4.10).

The paper is organized in the following way. In section 2, we recall basic facts on quantum $6 j$ symbols at roots of unity and the associated invariants of framed links following Kirillov and Reshetikhin [KR]. In section 3, we construct projective unitary representations of the mapping class groups and we prove our main results. In section 4 , we discuss a generalization of our construction for a complex simple Lie algebra based on the monodromy of the Wess-Zumino-Witten model.

Acknowledgement : The author would like to thank T. Kobayashi and M. Sakuma for helpful conversations on the tumnel number.

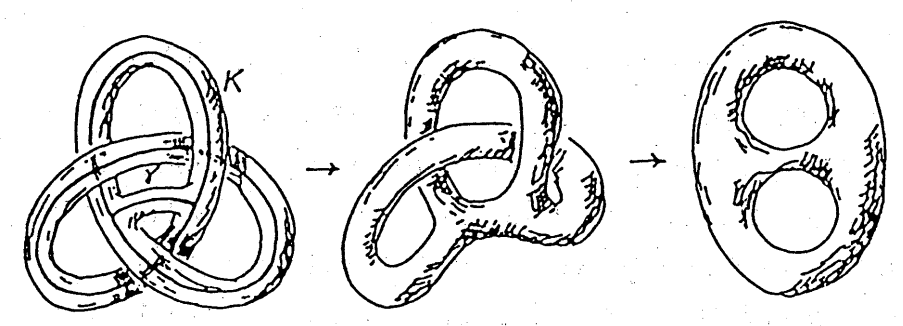

Figure 1 


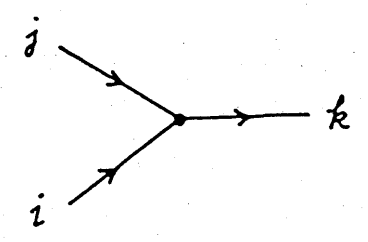

Figure 2

\section{Quantum $6 j$ symbols and tangle operators}

Let us recall an explicit form of quantum $6 j$-symbols at $q=e^{2 \pi \sqrt{-1} / r}$ following Killirov and Reshetikhin [KR] (see also [TV]).

We say that a triple $i, j, k \in P_{+}(l)$ is admissible if and only if it satisfies the conditions

$$
\begin{aligned}
& |i-j| \leq k \leq i+j \\
& i+j+k \in \mathrm{Z} \\
& i+j+k \leq l .
\end{aligned}
$$

We adopt the following convention of a $q$-integer.

$$
[n]=\frac{q^{n / 2}-q^{-n / 2}}{q^{1 / 2}-q^{-1 / 2}}=\frac{\sin n \pi / r}{\sin \pi / r} .
$$

For an admissible triple $(a, b, c)$ we put

$$
\Delta(a b c)=\left(\frac{[a+b-c] ![a+c-b] ![b+c-a] !}{[a+b+c+1] !}\right)^{1 / 2} .
$$

An ordered 6-tuple $a, b, c, d, e, f \in P_{+}(l)$ is said to be admissible if the triples

$$
(a, b, c),(c, d, e),(a, e, f),(b, d, f)
$$

are admissible in the above sense. For an admissible 6-tuple the Rakah-Wigner symbol is given by

$$
\begin{aligned}
& \left\{\begin{array}{lll}
a & b & c \\
d & e & f
\end{array}\right\}^{R W}=\Delta(a b c) \Delta(a e f) \Delta(b d f) \Delta(c d e) \sum_{z}(-1)^{z}[z+1] ! \times \\
& \{[z-a-b-c] ![z-a-e-f] ![z-b-d-f] ![z-c-d-e] ! \\
& \times[a+b+d+e-z] ![a+c+d+f-z] ![b+c+e+f-z] !\}^{-1}
\end{aligned}
$$

where the sum is for any non-negative integer $z$ such that all expressions in the brackets are non-negative. Normalizing the Rakah-Wigner symbol, the quantm $6 j$ symbol is given by

$$
\left\{\begin{array}{lll}
a & b & c \\
d & e & f
\end{array}\right\}=(-1)^{d+c+2 c-a-b} \sqrt{\frac{S_{0 c}}{S_{00}} \frac{S_{0 f}}{S_{00}}}\left\{\begin{array}{lll}
a & b & c \\
d & e & f
\end{array}\right\}^{R W}
$$


For an admissible triple $(i, j, k)$ and a trivalent graph depicted as in Figure 2, we associate a one dimensional complex vector space $\mathcal{H}_{i j}^{k}$ with basis denoted by $e_{i j}^{k}$. If $(i, j, k)$ is not admissible, we define $\mathcal{H}_{i j}^{k}$ to be zero. When we reverse the orientation of the edge corresponding to $i$ we use the convention that the same space is denoted by the symbol $\mathcal{H}_{i^{\dagger} j}^{k}$. We adopt a similar convention for $j$ and $k$.

By means of the quantum $6 j$-symbols we first define the fusing operators

$$
\begin{aligned}
& F_{+}: \oplus_{j} \mathcal{H}_{a b}^{j} \otimes \mathcal{H}_{j c}^{d} \rightarrow \oplus_{i} \mathcal{H}_{b c}^{i} \otimes \mathcal{H}_{a i}^{d} \\
& F_{-}: \oplus_{j} \mathcal{H}_{b c}^{j} \otimes \mathcal{H}_{a j}^{d} \rightarrow \oplus_{i} \mathcal{H}_{a b}^{i} \otimes \mathcal{H}_{i c}^{d}
\end{aligned}
$$

in the following way (see Figure 3)

$$
\begin{aligned}
& F_{+}\left(e_{a b}^{j} \otimes e_{j c}^{d}\right)=\sum_{i}\left\{\begin{array}{lll}
a & b & j \\
c & d & i
\end{array}\right\} e_{b c}^{i} \otimes e_{a i}^{d} \\
& F_{-}\left(e_{b c}^{j} \otimes e_{a j}^{d}\right)=\sum_{i}\left\{\begin{array}{lll}
c & b & j \\
a & d & i
\end{array}\right\} e_{a b}^{i} \otimes e_{i c}^{d} .
\end{aligned}
$$
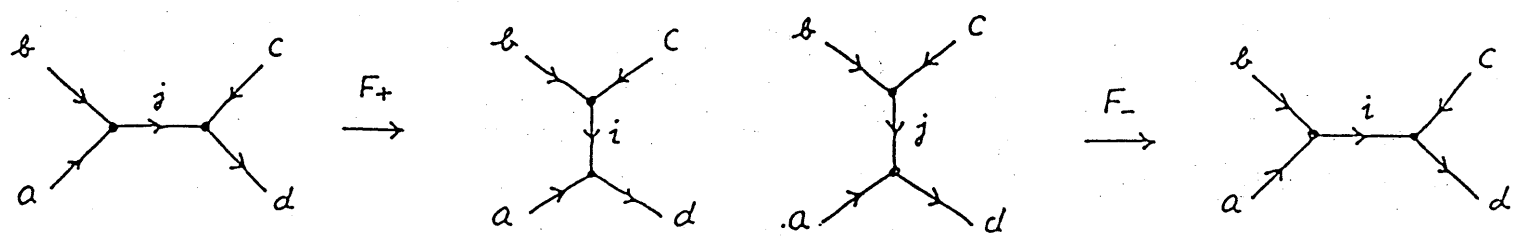

Figure 3

Let us introduce linear maps $\Omega_{+}$and $\Omega_{-}$

$$
\Omega_{ \pm}: \oplus_{j} \mathcal{H}_{j_{1} j_{2}}^{j} \rightarrow \oplus_{j} \mathcal{H}_{j_{2} j_{1}}^{j}
$$

given by

$$
\Omega_{ \pm}\left(e_{j_{1} j_{2}}^{j}\right)=(-1)^{j_{1}+j_{2}-j} \exp \left( \pm \pi \sqrt{-1}\left(\Delta_{j}-\Delta_{j_{1}}-\Delta_{j_{2}}\right)\right) e_{j_{2} j_{1}}^{j}
$$

We define the braiding operators

$$
B_{ \pm}: \oplus_{j} \mathcal{H}_{a b}^{j} \otimes \mathcal{H}_{j c}^{d} \rightarrow \oplus_{j} \mathcal{H}_{a c}^{j} \otimes \mathcal{H}_{j b}^{d}
$$

by

$$
B_{ \pm}=F_{-}\left(\Omega_{ \pm} \otimes i d\right) F_{+}
$$




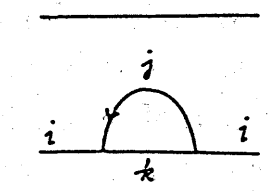

(a)

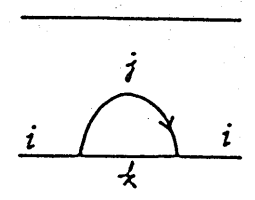

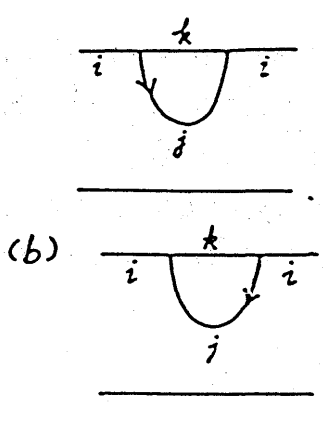

Figure 4

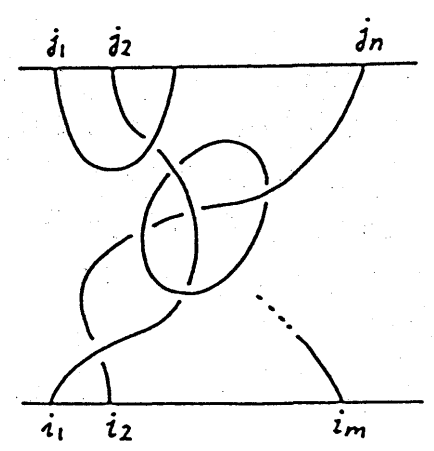

Figure 5

Thus we obtain the following commutative diagram.

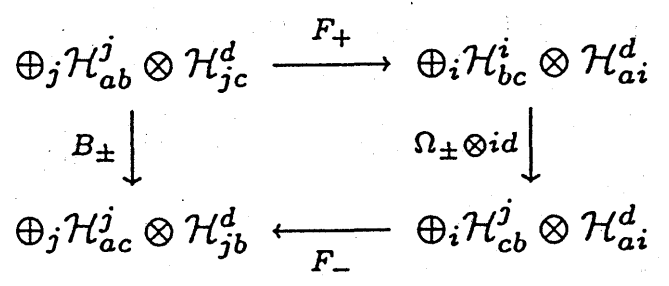

Associated with the diagram (a) in Figure 4, the fusing operators give the linear maps

$$
\begin{aligned}
& Y_{j^{\dagger}}^{0}: \oplus_{k} \mathcal{H}_{i j}^{k} \otimes \mathcal{H}_{k j^{\dagger}}^{i} \rightarrow \mathcal{H}_{j j^{\dagger}}^{0} \otimes \mathcal{H}_{i 0}^{i} \cong \mathrm{C} \\
& Y_{j^{\dagger} j}^{0}: \oplus_{k} \mathcal{H}_{i j^{\dagger}}^{k} \otimes \mathcal{H}_{k j}^{i} \rightarrow \mathcal{H}_{j^{\dagger} j}^{0} \otimes \mathcal{H}_{i 0}^{i} \cong \mathrm{C} .
\end{aligned}
$$

Normalizing these, we define the annihilation operators by

$$
\sqrt{S_{0 j} / S_{00} Y_{j j^{\dagger}}^{0}} \text { and }(-1)^{2 j} \sqrt{S_{0 j} / S_{00}} Y_{j^{\dagger} j}^{0}
$$

respectively. In a similar way, the fusion operators induce

$$
\begin{aligned}
& Y_{0}^{j j^{\dagger}}: \mathrm{C} \cong \mathcal{H}_{j j^{\dagger}}^{0} \otimes \mathcal{H}_{i 0}^{i} \rightarrow \oplus_{k} \mathcal{H}_{i j}^{k} \otimes \mathcal{H}_{k j^{\dagger}}^{i} \\
& Y_{0}^{j^{\dagger} j}: \mathrm{C} \cong \mathcal{H}_{j^{\dagger} j}^{0} \otimes \mathcal{H}_{i 0}^{i} \rightarrow \oplus_{k} \mathcal{H}_{i j^{\dagger}}^{k} \otimes \mathcal{H}_{k j}^{i}
\end{aligned}
$$

associated with the diagram (b) in Figure 4. Using this, we define the creation operators by

$$
\sqrt{S_{0 j} / S_{00}} Y_{0}^{j j^{\dagger}} \text { and }(-1)^{2 j} \sqrt{S_{0 j} / S_{00}} Y_{0}^{j^{\dagger} j}
$$

respectively.

We put

$$
\mathcal{H}_{j_{1} \cdots j_{n}}=\oplus_{k_{1}, \cdots, k_{n-1} \in P_{+}(l)} \mathcal{H}_{0 j_{1}}^{k_{1}} \otimes \mathcal{H}_{k_{1} j_{2}}^{k_{2}} \otimes \cdots \mathcal{H}_{k_{n-1} j_{n}}^{0}
$$


Let $T$ be an oriented framed $(m, n)$ tangle, by which we mean a union of oriented arcs in $\mathrm{R}^{2} \times[0,1]$ with boundary points contained in $\mathrm{R}^{2} \times\{0\} \cup \mathrm{R}^{2} \times\{1\}$ as shown in Figure 5. Here the framing is represented by the blackboard framing in the sense of [KM]. By decomposing the tangle diagram into elementary diagrams and by associating to each elementary diagram the braiding, creation or annihilation operator defined abore, we obtain a linear map.

$$
J_{r}(T): \mathcal{H}_{j_{1} \cdots j_{n}} \rightarrow \mathcal{H}_{i_{1} \ldots i_{m}}
$$

In particular, for an oriented framed link $L$ with $m$ components assigned with color $\lambda$ : $\{1, \cdots, m\} \rightarrow P_{+}(l)$ we obtain an invariant of colored oriented framed link $J_{\tau}(L, \lambda)$. It turns out that this invariant does not depend on the orientation on the link $L$. In the case $\lambda \equiv 1 / 2$, the invariant $J_{L}=J_{r}(L, 1 / 2)$ is characterized by the skein reation

$$
\begin{aligned}
& t J_{L_{+}}-t^{-1} J_{L_{-}}=\left(t^{2}-t^{-2}\right) J_{L_{0}} \\
& J_{O}=[2]=2 \cos \frac{\pi}{r}
\end{aligned}
$$

where we use the usual notation $L_{+}, L_{-}, L_{0}$ as in [Jo].

Let $R$ be the representation ring of $s l(2, \mathrm{C})$. The above invariant defines a linear map

$$
J_{r}(L,:): R^{\otimes m} \rightarrow \mathbf{C}
$$

Moreover, it factors through the fusion algebra $R_{r}$ for the $S U(2)$ Wess-Zumino- IIitten model at level $l=r+2$ (see [V]). This linear map is compatible with the product structure as shown in Figure 6. Here $R_{r}$ has generators $v_{j}, j \in P_{+}(l)$ with the product structure

$$
v_{i} \cdot v_{j}=\sum_{k} N_{i j}^{k} v_{k}
$$

where $N_{i j}^{k}=\operatorname{dim} \mathcal{H}_{i j}^{k}$ (see also [MoS1] and $[\mathrm{MoS} 2]$ ). As the notation indicates it is clear from the above consideration that the invariant $J_{r}(K, j)$ for a framed knot $K$ coincides with the one defined in the introduction by means of the recursive formula 1-3.

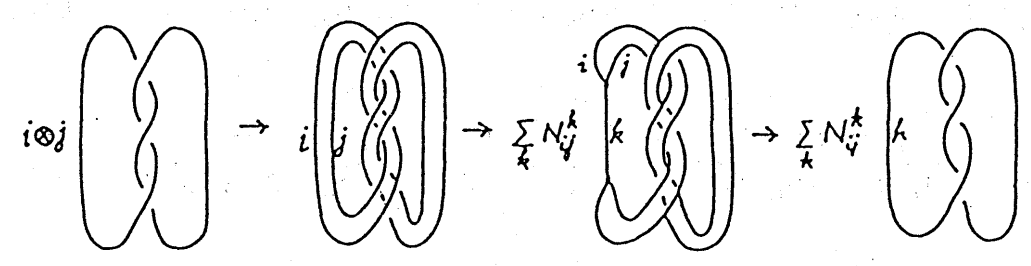

Figure 6 
Let us recall that we have the following (see $[\mathrm{KM}]$ ).

2.9 Proposition. Let $T$ be a colored oriented framed tangle. We write $J(T)$ for $J_{r}(T)$. Then, with respect to the following local modifications of the tangle we have

$$
\begin{aligned}
& { }^{J} \mathrm{O}^{j}=\frac{S_{0 j}}{S_{00}} J \\
& { }^{J} \alpha^{j}=\exp 2 \pi \sqrt{-1} \Delta_{j} J_{\left({ }^{j}\right.} \text {, } \\
& J\left(\bigcup_{j}^{i}=\frac{S_{i j}}{S_{0 i}} J\right)^{i} .
\end{aligned}
$$

Here $\Delta_{j}$ is given by

$$
\Delta_{j}=\frac{j(j+1)}{r}
$$

2.10 Remark. Let us note that the sign convention adopted here is different from the one in $[\mathrm{KR}]$ and $[\mathrm{MoS} 1]$, where the creation and annihilation operators are defined without the sign $(-1)^{2 j}$ and consequently the effect of local modifications in Proposition 2.9 appear with signs.

\section{Proof of main results}

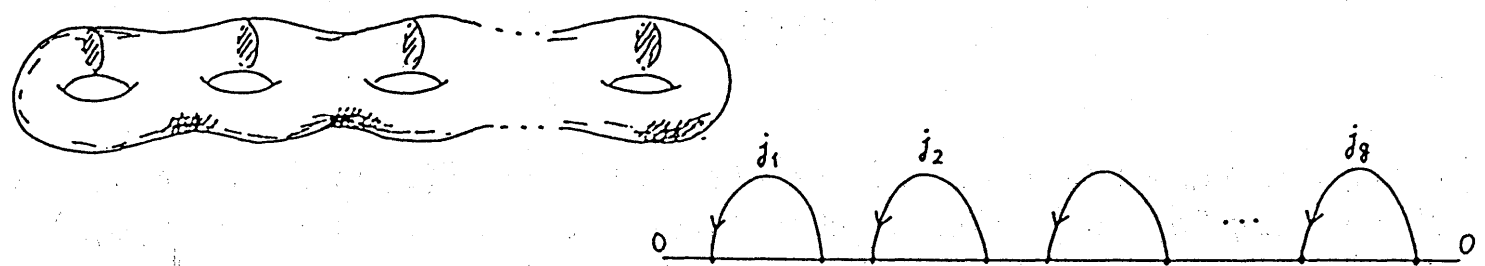

Figure 7

Let $\Sigma$ be a closed oriented surface of genus $g$. The mapping class group of $\Sigma$ denoted by $\mathcal{M}_{g}$ is by definition

$$
\mathcal{M}_{g}=\operatorname{Diff}^{+}(\Sigma) / \sim
$$

where $D$ iff $f^{+}(\Sigma)$ denotes the group of orientation preserving diffeomorphisms of $\Sigma$ and $\sim$ stands for the equivalence modulo isotopy. We are going to investigate an action of the mapping class group $\mathcal{M}_{g}$ on a vector space $\mathcal{H}_{\Sigma}$ defined by

$$
\mathcal{H}_{\Sigma}=\oplus_{j_{1}, \cdots, j_{g} \in P_{+}(l)} \mathcal{H}_{j_{1} j_{1}^{\dagger} \cdots j_{g} j_{g}^{\dagger}}
$$



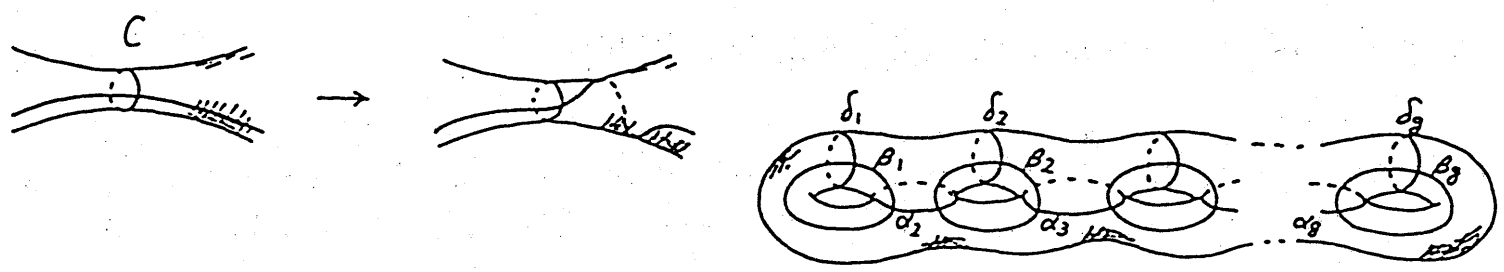

Figure 8

(see Figure 7).

Let us recall that the Dehn twist along a circle $C \subset \Sigma$ is a diffeomorphim of $\Sigma$ defined by cutting the surface $\Sigma$ along $C$ and by reglueing after the rotation of $2 \pi$ in the positive direction (see Figure 8). It is known by Lickorish [L] that $\mathcal{M}_{g}$ is generated by

$$
\alpha_{i}, \quad 2 \leq i \leq g ; \quad \beta_{i}, \quad 1 \leq i \leq g ; \quad \delta_{i}, \quad 1 \leq i \leq g
$$

which are Dehn twists along circles shown as in Figure 8.

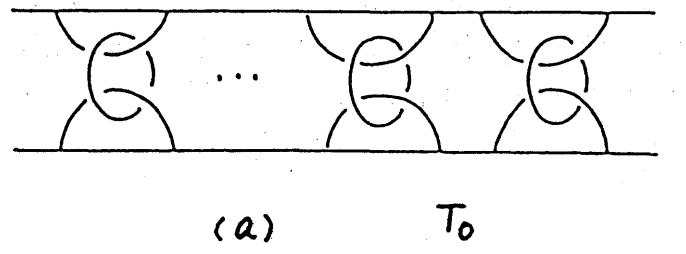

(a)

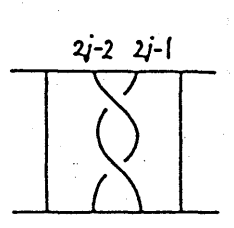

$T\left(\alpha_{j}\right)$

(b)

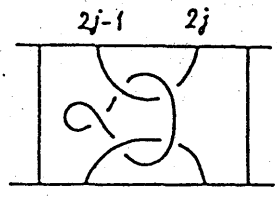

$T\left(\beta_{j}\right)$

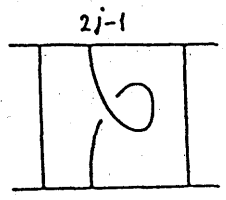

$T\left(\delta_{j}\right)$

Figure 9

To each $x \in \mathcal{M}_{g}$ we associate a framed $(2 g, 2 g)$ tangle $T(x)$ in the following way. Let $T_{0}$ be a $(2 g, 2 g)$ tangle illustrated in Figure 9a. To each $x_{i},(x=\alpha, \beta, \delta)$ we associate a $(2 g, 2 g)$ tangle $T\left(x_{i}\right)$ depicted as in Figure $9 \mathrm{~b}$ and for $x_{i}^{-1}$ we associate its mirror image $T\left(x_{i}^{-1}\right)$. Let $T_{0}$ be a $(2 g, 2 g)$ tangle shown in Figure 9. Given $x \in \mathcal{M}_{g}$ we express $x$ using Lickorish generators as $x_{i_{1}}^{\epsilon_{1}} \cdots x_{i_{k}}^{\epsilon_{k}}, \epsilon_{i}= \pm 1$ and we define a tangle $T(x)$ by the composition

$$
T(x)=T_{0} \circ T\left(x_{i_{1}}^{\epsilon_{1}}\right) \circ \cdots \circ T\left(x_{i_{k}}^{\epsilon_{k}}\right)
$$

Let $T(x)$ be an oriented framed $(2 g, 2 g)$ tangle defined for $x \in \mathcal{M}_{g}$ in the previous paragraph by fixing a way of writing $x$ in terms of Lickorish generators. We divide the set of arcs in $T(x)$ into $E^{0}(x), E^{1}(x)$ and $L(x)$ defined by

$$
\begin{aligned}
E^{0}(x) & =\left\{\gamma ; \partial \gamma \subset \mathrm{R}^{2} \times\{0\}\right\} \\
E^{1}(x) & =\left\{\gamma ; \partial \gamma \subset \mathrm{R}^{2} \times\{1\}\right\} \\
L(x) & =\{\gamma ; \partial \gamma=\phi\}
\end{aligned}
$$




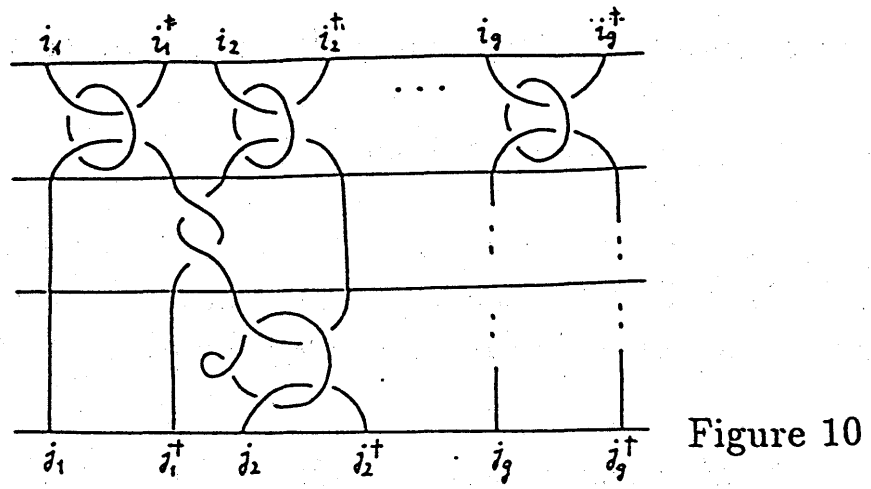

(see Figure 10).

We define a linear map

$$
\rho(x): \mathcal{H}_{\Sigma} \rightarrow \mathcal{H}_{\Sigma}
$$

in the following way. For

$$
u \in \mathcal{H}_{j_{1} j_{1}^{\dagger} \cdots j_{g} j_{g}^{\dagger}}, \quad v^{*} \in\left(\mathcal{H}_{i_{1} i_{1}^{\dagger} \cdots i_{g} i_{s}^{\dagger}}\right)^{*}
$$

we associate the colors to the arcs in $E^{0}(x)$ and $E^{1}(x)$ as shown in Figure 10.

As is explained above, each time we associate a color

$$
\lambda:\{1,2, \cdots, m\} \rightarrow P_{+}(l)
$$

to the components of the oriented framed link $L(x)=L_{1} \cup \cdots \cup L_{m}$ we have a linear map $J_{r}(T(x), \lambda): \mathcal{H}_{\Sigma} \rightarrow \mathcal{H}_{\Sigma}$. Let us denote by

$$
<\cdot,>: \mathcal{H}_{\Sigma}^{*} \times \mathcal{H}_{\Sigma} \rightarrow \mathrm{C}
$$

the canonical pairing with the dual space. We define $\rho(x)$ by

$$
\begin{aligned}
<v^{*}, \rho(x) u> & =\sqrt{S_{0 j_{1}} \cdots S_{0 j_{g}}} \sqrt{S_{0 i_{1}} \cdots S_{0 i_{g}}} \\
& \sum_{\lambda} C^{s i g n(L(x))} S_{0 \lambda(1)} \cdots S_{0 \lambda(m)}<v^{*}, J_{r}(T(x), \lambda) u>
\end{aligned}
$$

where $\lambda$ runs over all colors on the components of the link $L(x)$.

For $x, y \in \mathcal{M}_{g}$ we fix a way of writing $x$ and $y$ in terms of Lickorish generators and we define $(2 g, 2 g)$ tangles $T(x), T(y)$ and $T(x y)$ in the above way. Corresponding to this we have oriented framed links $L(x), L(y)$ and $L(x y)$. We put

$$
\sigma(x, y)=\operatorname{sign}(L(x y))-\operatorname{sign}(L(x))-\operatorname{sign}(L(y))
$$

and we define $\xi(x, y)$ by

$$
\xi(x, y)=C^{\sigma(x, y)}
$$


Let us recall that a closed oriented 3 -manifold $M$ is represented as

$$
M=V \cup_{h}(-V)
$$

with a handlebody $V$ and a glueing map $h: \partial V \rightarrow \partial V$. This description is called a Heegaard splitting. The minimal $g$ such that $M$ admits a Heegaard splitting with a handlebody of genus $g$ is called the Heegaard genus and is denoted by $g(M)$.

3.5 Proposition. The above construction defines a projectively linear representation

$$
\rho: \mathcal{M}_{g} \rightarrow G L\left(\mathcal{H}_{\Sigma}\right)
$$

with a 2-cocycle $\xi$. Namely, we have

$$
\rho(x y)=\xi(x, y) \rho(x) \rho(y)
$$

for any $x, y \in \mathcal{M}_{g}$. Moreover, with respect to a basis of $\mathcal{H}_{\Sigma}$

$$
e_{0 j_{1}}^{k_{1}} \otimes e_{k_{1} j_{1}^{\dagger}}^{k_{2}} \otimes \cdots \otimes e_{k_{2 g-1} j_{g}^{\dagger}}^{0}, \quad j_{1}, \cdots, j_{g}, k_{1}, \cdots k_{2 g-1} \in P_{+}(l)
$$

$\rho(x)$ is represented as a unitary matrix for any $x \in \mathcal{M}_{g}$.

Proof: To show the well-definedness of $\rho(h)$ for $h \in \mathcal{M}_{g}$ let us first recall that a key step to show the topological invariance of the expresstion 1.6 in [RT] is the following equality for the tangle operators corresponding to Kirby moves (see $[\mathrm{K}]$ and [FR]). For the tangle diagrams $\Gamma$ and $\Gamma^{\prime}$ as shown in Figure 11, we have

$$
C^{-1} \sum_{j} S_{0 j} J_{r}\left(\Gamma, i_{1}, \cdots, i_{n}, j\right)=J_{r}\left(\Gamma^{\prime}, i_{1}, \cdots, i_{n}\right)
$$

In our situation this equality can be applied to any local modification of the above type, allowing also the arcs in $E^{0}(h)$ and $E^{1}(h)$ as strands passing through the circle in the tangle diagram $\Gamma$.

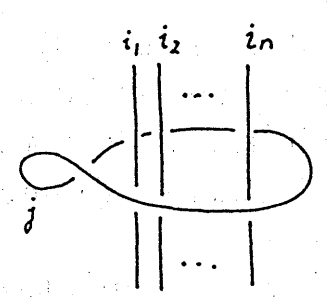

$r$

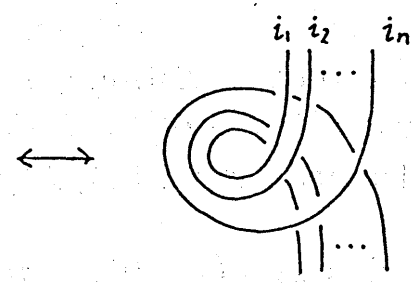

$r^{\prime}$

Figure 11 
Let us also recall that associated with a decomposition of $h$ into a product of Lickorish generators we can construct a link in $S^{3}$ so that the Dehn surgery on this link gives a 3-manifold obtained as a Heegaard splitting with a glueing map $h$ (see [Ro]). In our case the Dehn surgery on the link $L(h)$ gives a 3-manifold $V_{g} \cup_{h}\left(-V_{g}\right)$. The arcs in $E^{i}(h), i=0,1$ appear as a spine of the handlebody. Now we apply a relative version of Kirby's theorem (see $[\mathrm{K}]$ and [RT]) and we can conclude that if $h$ and $h^{\prime}$ are isotopic the associated tangle diagrams $T(h)$ and $T\left(h^{\prime}\right)$ are obtainable one from the other by a finitlely many steps of Kirby moves shown in Figure 11. Here we allow any arcs in the tangle diagram as strands passing through the circle. Hence the equality 3.6 implies $\rho(h)=\rho\left(h^{\prime}\right)$.

Now we describe the relation between $\rho(x y)$ and $\rho(x) \rho(y)$. First, let us notice that the linear operator $\rho\left(T_{0}\right)$ associated with the tangle $T_{0}$ is the identity operator. This can be shown by considering the composition $T_{0} \cdot T_{0}$ in the following way. Applying Kirby moves, one sees that $\rho\left(T_{0}\right)$ is idempotent. Since $\rho\left(T_{0}\right)$ is non-singular, this implies that $\rho\left(T_{0}\right)$ is identity. Combining with the definition 3.3 , it is easy to deduce the assertion $\rho(x y)=\xi(x, y) \rho(x) \rho(y)$.

Noticing that the entries of the fusing matrices are real at $q=e^{2 \pi \sqrt{-1}} / r$, it follows from the orthogonality of the quantum $6 j$ symbol (see [KR] and [RT]) that the braiding operator is represented by a unitary matrix with respect to the above basis. The unitarity of $\rho\left(x_{i}\right)$ for the Lickorish generators follows immediately by looking at the above tangle representation. This completes the proof.

Now we are in a position to prove our main results. Let $M$ be a closed oriented 3-manifold and let $K$ be a knot in $M$. We denote by $E(K)$ the knot exterior. Namely, $E(K)$ is the closure of the complementary space of the tubular neighborhood $N(K)$. If the tunnel number of the knot $K$ is $t(K)$, then we have a splitting of the knot exterior

$$
E(K)=\operatorname{cl}(V-N(K)) \cup_{h}(-V)
$$

with a handlebody $V$ of genus $g=t(K)+1$ and a glueing map $h: \partial V \rightarrow \partial V$. Here the knot $K$ is embedded in a simple position as shown in Figure 12. More precisely, there exists a separating disc $D$ properly embedded in $V$ with $\partial D \subset V$ such that $K$ is embedded as a core of the solid torus obtained by cutting the handlebody $V$ along $D$. Let us note that $c l(V-N(K))$ is a special case of a so-called compression body.

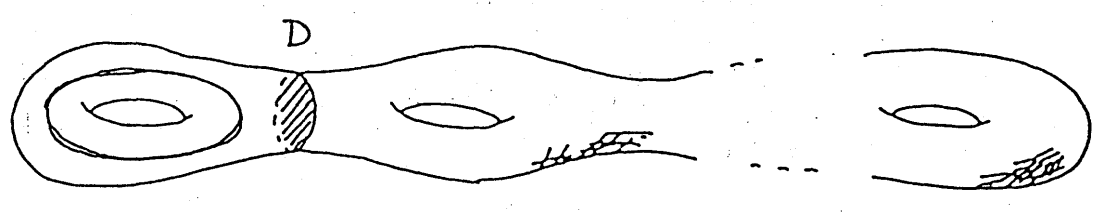

Figure 12 
We put

$$
v_{j}=e_{0 j}^{j} \otimes e_{j j^{+}}^{0} \otimes e_{00}^{0} \otimes \cdots \otimes e_{00}^{0}
$$

which is a basis of

$$
\mathcal{H}_{0 j}^{j} \otimes \mathcal{H}_{j j^{\dagger}}^{0} \otimes \mathcal{H}_{00}^{0} \otimes \cdots \otimes \mathcal{H}_{00}^{0} \cong \mathbf{C}
$$

With this notation we obtain the following.

3.7 Proposition. In the above situation we have

$$
Z_{r}(M, K ; j)=S_{00}^{-g}<v_{j}^{*}, \rho(h) v_{0}>
$$

Proof: Let us close the $(2 g, 2 g)$ tangle diagram $T(h)$ to get a plat diagram as in Figure 13. This is a link diagram expressed as $L(h) \cup L$ where $L$ contains $2 g$ components $L_{1}, \cdots, L_{2 g}$. The color on $L_{1}$ is equal to $j$ and the color on the other components is zero. Let us recall that by the Dehn surgery on the framed link $L(h)$ we get our 3-manifold $M$ with link $L$ in it.

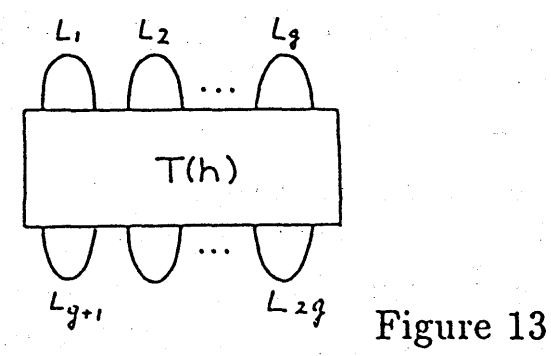

Let us consider the above situation with $K=L_{1}$. Noticing that the fusion operator

$$
Y_{j j^{\dagger}}^{0}: \mathrm{C} \cong \mathcal{H}_{0 j}^{j} \otimes \mathcal{H}_{j^{\dagger}}^{0} \rightarrow \mathcal{H}_{j^{\dagger}}^{0} \otimes \mathcal{H}_{00}^{0} \cong \mathrm{C}
$$

is an identity map, we obtain the assertion from definition 1.5 and 3.3 .

Now the proof of our main results is completed in the following way. We obtain from Proposition 3.5 that $\left\langle v_{j}^{*}, \rho(h) v_{0}\right\rangle$ is an entry of a unitary matrix. Hence it follows that

$$
\sum_{j \in P_{+}(l)}\left|<v_{j}^{*}, \rho(h) v_{0}>\right|^{2} \leq 1,
$$

which shows Theorem B. In the case $M=S^{3}$ we have

$$
Z_{r}\left(S^{3}, K ; j\right)=J_{r}(K, j)
$$


Moreover, by means of the symmetry principle due to Kirby and Melvin [KM], we have

$$
\left|J_{r}(K, j)=J_{r}\left(L_{1} \cup L_{2} \cup \cdots L_{g}, \lambda\right)\right|
$$

for $\lambda(1)=j$ and

$$
\lambda(k)=0 \text { or } l / 2, \quad 2 \leq k \leq g .
$$

Combining this symmetry with Theorem B, we obtain Theorem A. This completes the proof.

\section{Comments on generalizations and discussions}

We discuss briefly how our results can be generalized to an arbitrary complex simple Lie algebra $\mathcal{G}$. We refer the readers to [Ko3] for a more detailed description. We fix a positive integer $l$ and we adopt as the set of colors

$$
P_{+}(l)=\left\{\lambda \in P_{+} ; 0 \leq(\lambda, \theta) \leq l\right\}
$$

where $(\cdot, \cdot)$ is the Cartan-Killing form normalized so that $(\theta, \theta)=2$ for the longest root. Let $L$ be an oriented framed link in $S^{3}$. By looking at the monodromy representation of the Knizhinik-Zamolodchikov equation in Wess-Zumino-Witten model at level $l$ (see $[\mathrm{TK}]$ ), for a color $\lambda:\{1, \cdots, m\} \rightarrow P_{+}(l)$, one can define an invariant of colored framed link $J_{\mathcal{G}, r}(L, \lambda)$ where we put $r=l+h^{*}$ with the dual Coxeter number $h^{*}$.

The phase factor derived from the change of framing in Proposition 2.9 is expressed by the conformal weight

$$
\Delta_{\lambda}=\frac{(\lambda, \lambda+2 \rho)}{2\left(l+h^{*}\right)}
$$

where $\rho$ is the half sum of the positive roots.

Now in the situation of Theorem B, the Witten invariant for $\mathcal{G}$ at level $l$ is expressed as

$$
Z_{\mathcal{G}, r}(M, K, \lambda)=C^{s i g n(L)} \sum_{\mu} S_{0 \mu(1)} \cdots S_{0 \mu(m)} J_{\dot{\mathcal{G}, r}}(K \cup L, \lambda \cup \mu)
$$

where the sum is for any $\mu:\{1, \cdots, m\} \rightarrow P_{+}(l)$. In the above expression $C$ and $S_{0 \lambda}$ are given by

$$
\begin{aligned}
& C=\exp \left(-\frac{l \operatorname{dim} \mathcal{G} \pi \sqrt{-1}}{4\left(l+h^{*}\right)}\right) \\
& S_{0 \lambda}=\frac{1}{\left(l+h^{*}\right)^{\operatorname{rank}(\mathcal{G}) / 2}}\left(\frac{\operatorname{vol} \Lambda^{W}}{\operatorname{vol} \Lambda^{R}}\right)^{1 / 2} \prod_{\alpha \in \Delta_{+}} 2 \sin \frac{\pi(\lambda+\rho, \alpha)}{l+h^{*}} .
\end{aligned}
$$

Here vol $\Lambda^{W}$ is the volume of the weight lattice, vol $\Lambda^{R}$ is the volume of the coroot lattice, and $\Delta_{+}$stands for the set of positive roots. Let us notice that $C$ can be 
कritten as $\left(\exp 2 \pi \sqrt{-1} \frac{c}{24}\right)^{-3}$ with $c$ the central charge of the Virasoro algebra acting on the integrable highest weight module of highest weight $\lambda$. We also recall that $S_{0 \lambda}$ appears as an entry of the modular transformation $S$ matrix on the characters of level : integrable highest weight modules determined in $[\mathrm{KP}]$ and $[\mathrm{KW}]$.

Several approaches have been developed to establish a more combinatorial descripín of such invariants for classical Lie algebras (see [M], [TW], [We] and [KT]). The sssociated representation of the mapping class group is related to the monodromy zepresentation of the conformal field theory on Riemann surfaces (see [TUY]).

Let $K$ be a knot in a closed oriented 3-manifold $M$. Generalizing Theorem B, we ¿ave a lower estimate for the tunnel number $t(K)$

$$
\sum_{\lambda \in P_{+}(l)}\left|Z_{\mathcal{G}, r}(M, K ; \lambda)\right|^{2} \leq S_{00}^{-2(t(K)+1)} .
$$

Te also notice that considering the case $K$ is empty, we have the following lower sound for the Heegaard genus (see [Wa], [Ko2] and [G]).

$$
\left|Z_{\mathcal{G}, r}(M)\right| \leq S_{00}^{-g(M)}
$$

Let us discuss the case $\mathcal{G}=\operatorname{sl}(n, \mathrm{C})$. We denote by $\alpha_{i}, 1 \leq i \leq n-1$, a set of simple zoots and $s_{\alpha_{i}}$ the reflection on the weight lattice defined by $s_{\alpha_{i}}(v)=v-\left(v, \alpha_{i}\right) \alpha_{i}$. The set of weights $P_{+}(l)$ is written as

$$
P_{+}(l)=\left\{\sum_{i=1}^{n-1} a_{i} \Lambda_{i} ; a_{i} \in \mathrm{Z}, a_{i} \geq 0, \sum_{i=1}^{n-1} a_{i} \leq l\right\}
$$

*ith the fundamental weights $\Lambda_{i}, 1 \leq i \leq n-1$, which is naturally identified with the Fit of dominant integral weights for the corresponding affine Lie algebra $\widehat{\mathcal{G}}$ at level $\therefore$ Hence we have a Dynkin diagram automorphism for the affine Lie algebra, which =duces an automorphism on the set $P_{+}(l)$. This automorphism is isomorphic to a zclic group of order $n$ generated by $\sigma$ defined by

$$
\sigma(\lambda)=\mathcal{C}(\lambda)+l \Lambda_{1}
$$

- jere $\mathcal{C}$ is the Coxeter transformation $s_{\alpha_{1}} \cdots s_{\alpha_{n-1}}$. In $[\mathrm{KT}]$ we described the phase Ector coming from this action, which implies that for a knot $K$ in $S^{3}$ we have

$$
\left|J_{\mathcal{G}, r}(K, \sigma(\lambda))\right|=\left|J_{\mathcal{G}, r}(K, \lambda)\right| \text {. }
$$

Let us notice that $\sigma$ has a fixed point $l / n \sum_{i=1}^{n-1} \Lambda_{i}$ if $l \equiv 0 \bmod n$ and is fixed point Ze otherwise. We suppose that $\lambda \in P_{+}(l)$ is not a fixed point of $\sigma$ and that $\lambda$ is not ntained in the orbit of $0 \in P_{+}(l)$. Then, we have an inequality

$$
\sum_{\lambda \in P_{+}(l)}\left|J_{\mathcal{G}, r}(K, \lambda)\right|^{2} \leq n^{-t(K)} S_{00}^{-2(t(K)+1)}
$$


We recall that the skein (or HOMFLY) polynomial $P_{L}(a, z)$ for an oriented link is a two-variable generalization of the Jones polynomial (see [Jo]) characterized by the skein relation:

$$
\begin{aligned}
& P_{O}=1 \\
& a^{-1} P_{L_{+}}-a P_{L_{-}}=z P_{L_{0}} .
\end{aligned}
$$

Let us suppose that $n \geq 3$ and $l \geq 2$. Considering the case when $\lambda \in P_{+}(l)$ corresponds to the vector representation, we have

$$
P_{K}\left(s^{n}, s-s^{-1}\right)=\frac{\sin \pi / r}{\sin n \pi / r} J_{\mathcal{G}, r}(K, \lambda)
$$

for $s=e^{\pi \sqrt{-1} / r}$. Combining with 4.6 and 4.7, we obtain a upper bound for the above special values of the skein polynomial. A similar estimate can be formulated for special values of the Kauffman polynomial by examining the case $\mathcal{G}=o(n, \mathbf{C}), s p(n, \mathbf{C})$.

Our construction may also be generalized to certain links. Let $L=L_{1} \cup L_{2}$ be a link with 2 components in a closed oriented 3-manifold $M$. We define its tunnel number $t(L)$ to be the minimal number $g+1$ such that the link exterior $E(L)=\operatorname{cl}(M-N(L))$ admits a splitting

$$
E(L)=\left(V^{1}-N\left(L_{1}\right)\right) \cup\left(V^{2}-N\left(L_{2}\right)\right)
$$

with a handlebody $V^{1}$ of genus $g$ and its second copy $V^{2}$ with a reversed orientation. Here we assume that $L_{i}, i=1,2$, is embedded in $V^{i}$ in a simple position as shown in Figure 12. In this situation we obtain for $\lambda_{1} \in P_{+}(l)$

$$
\sum_{\lambda_{2} \in P_{+}(l)}\left|Z_{\mathcal{G}, r}\left(M, L ; \lambda_{1}, \lambda_{2}\right)\right|^{2} \leq S_{00}^{-2(t(L)+1)}
$$

where $\lambda_{i}$ is a color on $L_{i}, i=1,2$.

\subsection{Concluding Remarks.}

(i) It was shown by Jones [Jo] that if an oriented link $L$ is a closed $n$ braid, then

$$
\left|V_{L}\left(e^{2 \pi \sqrt{-1} / r}\right)\right| \leq\left(2 \cos \frac{\pi}{r}\right)^{n-1}, \quad r=3,4, \cdots
$$

The unitarity of the monodromy representation enables us to generalize this inequality in the form

$$
\left|J_{\mathcal{G}, r}(L, \lambda)\right| \leq\left(\frac{S_{0 \lambda}}{S_{00}}\right)^{n}
$$

using the framed link invariant with color $\lambda \in P_{+}(l)$ for each component of $L$. Combining with our estimate of the tunnel number, we can deduce several consequences. 
For example, if a knot $K$ satisfies the equality for $r=5$ and $n=3$ in 4.11 (see [Jo]), then we have $t(K) \geq 2$.

(ii) For a connected sum of knots we have

$$
J_{\mathcal{G}, r}\left(K_{1} \sharp K_{2}, \lambda\right)=J_{\mathcal{G}, r}\left(K_{1}, \lambda\right) J_{\mathcal{G}, r}\left(K_{2}, \lambda\right) \frac{S_{00}}{S_{0 \lambda}} .
$$

For a knot $K$, let us consider $K \sharp \cdots \sharp K$ ( $n$ times). Our result can be used to give a lower estimate for the tunnel number of such knot in terms of a function of $n$ which is of linear order with respect to $n$.

\section{REFERENCES}

[CLM] S. Cappell, R. Lee and E. Y. Miller, Invariants of 3-manifolds from conformal field theory, Topology '90, eds. B. Apanasov et al (1992), 91-110.

[C] L. Crane, 2d physics and 3d topology, Commun. Math. Phys 135 (1991), 615-640.

[D] V. G. Drinfel'd, Quantum groups, Proc. Int. Cong. Math. Berkeley (1987), 798-820.

[FR] R. Fenn and C. Rourke, On Kirby's calculus of links, Topology 18 (1979), 1-15.

[G] S. Garoufalidis, Relations among 3-manifold invariants, Thesis, The University of Chicago, 1992.

[J] M. Jimbo, A q-difference analogue of $U(\mathcal{G})$ and the Yang-Baxter equation, Letters in Math. Phys 10 (1985), 63-69.

[Jo] V. F. R. Jones, Hecke algebra representations of braid groups and link polynomials, Ann. of Math 126 (1987), 355-388.

[KP] V. G. Kac and D. H. Peterson, Infinite dimensional Lie algebras, theta functions and miodular forms, Adv. in Math 53 (1984), 125-264.

[KW] V. G. Kac and M. Wakimoto, Modular and conformal invariance in the representation theory of affine Lie algebras, Adv. in Math 40 (1988), 156-236.

[Ki] R. Kirby, A calculus for framed links, Invent. Math 45 (1978), 35-56.

[KM] R. Kirby and P. Melvin, The 3-manifold invariants of Witten and Reshetikhin-Turaev for sl $(2, \mathrm{C})$, Invent. Math 105 (1991), 473-545.

[KR] A. N. Kirillov and N. Y. Reshetikhin, Representations of the algebra $U_{q}(s l(2))$, q-orthogonal polynomials and invariants of links, Infinite dimensional Lie algebras, ed. V. G. Kac, World Scientific (1988), 285-339.

[Ko1] T. Kohno, Topological invariants for 3-manifolds using representations of mapping class groups I, Topology 31 (1992), 203-230.

[Ko2] T. Kohno, Three-manifold invariants derived from conformal field theory and projective representations of modular groups, Int. J. Mod. Phys. B 6 (1992), 1795-1805.

[Ko3] T. Kohno, Topological invariants for 3-manifolds using representations of mapping class groups II: Estimating tunnel number of knots, preprint 1992, submitted to Contemp. Math.

[KT] T. Kohno and T. Takata, Symmetry of Witten's 3-manifold invariants for sl(n, C), to appcar in Journal of Knot Theory and Its Ramifications.

[L] W. B. R. Lickorish, A finite generators for the homeotopy group of a 2-manifold, Proc. Camb. Phil. Soc 60 (1964), 769-778 (ibid. 62 (1966) 679-681).

[LM] W. B. R. Lickorish and K. Millet, Some evaluations of link polynomials, Comment. Math. Helv 61 (1986), 349-359.

[Lp] A. S. Lipson, An evaluation of a link polynomial, Math. Proc. Camb. Phil. Soc. 100 (1986), 361-364. 
[MS] G. Moore and N. Seiberg, Classical and quantum conformal field theory, Commun. Math. Phys 123 (1989), 177-254.

[M] H. R. Morton, Invariants of links and 3-manifolds from skein theory and from quantum groups, preprint, 1992.

[MoS1] H. R. Morton and P. M. Strickland, Jones polynomial invariants for knots and satellites, Math. Proc. Camb. Phil. Soc. 109 (1991), 83-103.

[MoS2] H. R. Morton and P. M. Strickland, Satellites and surgery invariants, preprint, 1990.

[P] S. Piunikhin, Reshetikhin-Turaev and Kontsevich-Kohno-Crane 3-manifold invariants coincide, preprint.

[RT] N. Y. Reshetikhin and V. G. Turaev, Invariants of 3-manifolds via link polynomials and quantum groups, Invent. Math 103 (1991), 547-597.

[Ro] D. Rolfsen, "Knots and Links," Publish or Perish Press, 1976.

[TK] A. Tsuchiya and Y. Kanie, Vertex operators in conformal field theory on $P^{1}$ and monodromy representations of braid groups, Advanced Studies in Pure Math 16 (1988), 297-372.

[TUY] A. Tsuchiya, K. Ueno and Y. Yamada, Conformal field theory on universal family of stable curves with gauge symmetries, Advanced Studies in Pure Math 19 (1989), 459-566.

[TV] V. G. Turaev and O. Y. Viro, State sum invariants of 3-manifolds and quantum $6 j$-symbols, LOMI preprint, 1990.

[TW] V. G. Turaev and H. Wenzl, Quantum invariants of 3-manifolds associated with classical simple Lie algebras, preprint, 1991.

[V] E. Verlinde, Fusion rules and modular transformations in 2-D conformal field theory, Nucl. Phys B300 (1988), 360-376.

[Wa] K. Walker, On Witten's 3-manifold invariants (and talk given at KNOTS 90, Osaka), preprint.

[We] H. Wenzl, Braids and invariants of 3-manifolds, preprint, 1992.

[W] E. Witten, Quantum field theory and the Jones polynomial, Commun. Math. Phys 121 (1989), 351-399.

E-mail address: t-kohno@tansei.cc.u-tokyo.ac.jp 